\title{
Effects of Lorazepam on the Neuromagnetic Mismatch Negativity (MMNm) and Auditory Evoked Field Component $\mathrm{NIOOm}$
}

\author{
Timm Rosburg*, 1,2,3, Varvara Marinou', Jens Haueisen², Stefan Smesny' and Heinrich Sauer' \\ 'Department of Psychiatry, Friedrich-Schiller-University Jena, Jena, Germany; ${ }^{2}$ Biomagnetic Center, Department of Neurology, Friedrich-Schiller- \\ University Jena, Jena, Germany; ${ }^{3}$ Department of Epileptology, University of Bonn, Bonn, Germany
}

\begin{abstract}
The mismatch negativity (MMN) as an auditory evoked potential is thought to reflect an early, preconscious attention process. While this component has gained great importance in studies on clinical populations and in basic research on auditory information processing, the involvement of different neurotransmitters in the generation of this component is less well understood. We investigated the impact of the benzodiazepine lorazepam as a GABA agonist on the neuromagnetic MMN (MMNm) and auditory evoked field component N I00m. A group of 12 healthy subjects was studied in single blind trials under the following three conditions: after the intake of $1.25 \mathrm{mg}$ lorazepam, $100 \mathrm{mg}$ caffeine or placebo. Neuromagnetic recordings were obtained before drug intake and three times after it. Controlled visual attention was tested additionally using a version of the Continuous Performance Test (CPT). The neuromagnetic activity was reconstructed by a single moving dipole, and the dipole moment and its latency were compared between conditions and time points of measurement. Lorazepam diminished the signal detection performance in the CPT 25 min after drug intake. The source of the field component $\mathrm{NIO0m}$ was attenuated, most significantly in the recording $105 \mathrm{~min}$ after lorazepam intake. The attenuation of the MMNm under lorazepam became significant at 105 min, but was visually less apparent, because in all conditions a decrease of the MMNm dipole moment within the course of a session was observed. Besides the already known effects of benzodiazepines on controlled attention functions, preconscious attention functions as reflected in the MMN are impaired by acute benzodiazepine intake. MMN studies on clinical populations have to be controlled for the recording time because of the strong habituation of this component. Neuropsychopharmacology (2004) 29, I723-1733, advance online publication, 5 May 2004; doi: I 0. I038/sj.npp. 1300477
\end{abstract}

Keywords: auditory cortex, auditory evoked potentials, attention, magnetoencephalography

\section{INTRODUCTION}

Evoked potentials are a powerful tool to investigate normal and disturbed brain function. The mismatch negativity (MMN) represents a component of auditory evoked potentials (AEP), reflecting the cortical response on a rare and discernible deviance in an acoustic stimulation. This deviance can refer to simple characteristics of the stimuli, as for example, tone pitch, stimulus intensity, or duration, and also to more complex characteristics as tone patterns or melodies. The MMN is calculated as the difference wave between the AEP of the deviant and standard tones. It is characterized as a fronto-central negativity in the time

\footnotetext{
*Correspondence: Dr T Rosburg, Department of Epileptology, University of Bonn, Sigmund-Freud-Str. 25, D-53105 Bonn, Germany, Tel: + 49228287 9346; Fax: + 492282876294 ,

E-mail: timm.rosburg@ukb.uni-bonn.de

Received 24 September 2003; revised 24 March 2004; accepted 29 March 2004

Online publication: 6 April 2004 at http://www.acnp.org/citations/ Npp04060403440/default.pdf
}

range of about $100-250 \mathrm{~ms}$. The MMN is thought to be the result of an automatic comparison process between the stored information on the standard stimulus and the incoming information on the deviant tone (for an overview, see Näätänen, 2001). Part of the clinical interest on this component stems from the fact that an MMN can be elicited even in the absence of directed attention, although recent studies on dichotic listening have also indicated a modulation of the MMN by directed attention (Alain and Woods, 1997; Woldorff et al, 1998).

The detection of discontinuities in the acoustic input represents a basic and early step in auditory information processing. In neuropsychiatric research, the interest in information processes is caused by the fact that cognitive deficits are crucial in many neurologic and psychiatric diseases. As a clear, comprehensive, and differential description of MMN deficits can be very helpful for the elucidation of pathogenetic processes, MMN has been investigated in various diseases, as for example, in Alzheimer's disease (for overview Pekkonen, 2000), in dyslexia (for an overview, see Kujala and Näätänen, 2001), 
or in alcoholism (for an overview, see Ahveninen et al, 2000). A reduced MMN amplitude in schizophrenia seems to represent one of the most consistent findings (for an overview, see Michie et al, 2000), pointing towards the notion of a disturbed early attention process in this disease.

The study of drug effects within this context is important for at least two reasons: first, deficits in MMN generation can be modeled by drug administration. The NMDA antagonist ketamine for instance leads to an attenuation of the MMN, which is similar to that observed in schizophrenia, and induces disturbances in perception and cognitive functioning, resembling symptoms observed in this disease (Umbricht et al, 2000; KreitschmannAndermahr et al, 2001; Umbricht et al, 2002). On contrast, MMN was unaffected by the intake of the $5-\mathrm{HT}_{2 \mathrm{~A}}$ receptor agonist psilocybin, although psilocybin induces cognitive disturbances and models other psychotic behavior (Umbricht et al, 2003). Similarly, tryptophan depletion leading to generally decreased 5-HT levels affected only the latency of the neuromagnetic MMN (MMNm) but not its amplitude (Ahveninen et al, 2002). A single dose of the dopamine $\mathrm{D}_{2}$ receptor antagonist haloperidol accelerated the MMNm to frequency change (Pekkonen et al, 2002), but the MMNm amplitude was not diminished (Kähkönen et al, 2001, 2002; Pekkonen et al, 2002). Other substances investigated in their effect on MMN were the cholinergic antagonist scopolamine (Pekkonen et al, 2001), the cholinesterase inhibitor tetrahydroaminoacridine (Riekkinen et al, 1997), the adrenocorticotropine fragment ACTH4-10 (Smolnik et al, 1999) or the histamine H1-receptor antagonist chlorpheniramine (Serra et al, 1996). With some precaution, a glutamatergic and cholinergic modulation of the MMN amplitude and a dopaminergic and serotonergic modulation of the MMN latency can be assumed on the basis of recent studies. However, direct and indirect drug action can so far hardly be differentiated.

The second reason for the investigation of drug effects is to rule out that observed group differences are associated with drug treatment. It can be concluded from recent studies that antipsychotics have no major impact on the finding of a reduced MMN in patients with schizophrenia (Catts et al, 1995; Javitt et al, 1995; Umbricht et al, 1998, 1999). In contrast to antipsychotic drugs, benzodiazepines have not been systematically investigated in their functional impact on MMN generation as yet, although they play an important role in psychiatric treatment and are commonly applied as comedication in acute psychotic patients. In general, benzodiazepines can serve as sedative-hypnotic, anxiolytic, muscle relaxing, or anticonvulsant medication. Their mechanism of action is to enhance GABAergic transmission. Benzodiazepines increase the probability of channel opening in response to GABA, thus accounting for their pharmacological and therapeutic actions (Study and Barker, 1981).

Nakagome et al (1998) investigated the impact of $0.25 \mathrm{mg}$ triazolam on MMN on the morning following a nocturnal administration and found an attenuation of the MMN. Unfortunately, that finding is restrained by methodological shortcomings, namely a fixed order of drug application (placebo condition always first) and the long latency between drug intake and MMN measurements. Smolnik et al (1998) examined whether the benzodiazepine antago- nist flumazenil affects the MMN and found an amplitude increase with flumazenil. While both findings point with some reservations to a modulating influence of GABA receptor activity on MMN generation, with benzodiazepines decreasing and benzodiazepine antagonists possibly increasing MMN amplitude, a recent study found no difference in MMN between schizophrenic patients treated with or without benzodiazepines (Murakami et al, 2002). Similarly, no difference in MMN was revealed between schizophrenic patients treated with high vs those with low doses of anxiolytic and hypnotic drugs, including benzodiazepines (Kasai et al, 2002). A reduction of the MMN by benzodiazepines seems to be, nevertheless, likely to some extent, since a diminution of event-related components caused by benzodiazepines was found in several studies, as for the N100 (Rockstroh et al, 1991; Semlitsch et al, 1995), P200 (Semlitsch et al, 1995), and P300 (Milligan et al, 1989; Nichols and Martin 1993; Rockstroh et al, 1991; Semlitsch et al, 1995).

Besides that, on the behavioral level an attenuation of attention performance with benzodiazepines is well documented (Duka et al, 1995; Fluck et al, 2001; Krystal et al, 1998; Micallef et al, 2001; Post et al, 1997). However, since attention can be regarded as a process of information and response selection, pure behavioral studies can hardly reveal which levels of information processing are affected by benzodiazepines. Only the use of electrophysiology offers the possibility to dissociate between early (more automatic) and later (more effort demanding) attention processes. The attenuation of the P300 after benzodiazepine intake can be regarded as evidence that later (more effort demanding) attention processes are influenced by the drug, while the impact on early (more automatic attention) processes, as reflected by the MMN, is still a matter of debate.

The main aim of the current single-blind study was to investigate the impact of the benzodiazepine lorazepam on the MMN in order to elucidate the GABAergic modulation of early auditory attention processes. We focused on the supratemporal MMN generator by applying MEG, which is highly selective for tangential sources. The supratemporal generator gives also the largest contribution to the MMN measured by EEG (Rinne et al, 2000; Waberski et al, 2001). Besides the $\mathrm{MMNm}$, the $\mathrm{N100m}$ was analyzed as an indicator for the obligatory sensory stimuli processing. Owing to the technical limitations of the available MEG system, only the left hemispheric N100m and MMNm were recorded. Lorazepam, an intermediate-acting benzodiazepine was applied as active drug. The quite common use in German psychiatric institutions as acute anxiolytic medication in psychotic patients made it well suited for study purposes. According to clinical experience, a dose of $1.25 \mathrm{mg}$ lorazepam was regarded as high enough to observe effects on behavior without causing to much drowsiness.

A placebo and caffeine condition were introduced for comparison. Two considerations led us to the inclusion of a caffeine condition. Firstly, an additional condition increased the subject's uncertainty about the applied drug. Secondly, a response decrease was expected to occur over time even in the placebo condition and caffeine was thought to counteract this response decrease. A decrease of the $\mathrm{N} 100 / \mathrm{N} 100 \mathrm{~m}$ amplitude as a result of ongoing stimulation is well described in electroencephalography (EEG) and 
magnetoencephalography (MEG) studies (EEG: May et al, 1994; Picton et al, 1976; Roeser and Price, 1969; MEG: Rosburg et al, 2000, 2002). Studies on MMN have revealed so far equivocal results. May et al (1994) observed no alteration of the MMN over time, while a clear decrease of the MMN amplitude was found in the study of McGee et al (2001) already after $10 \mathrm{~min}$ recording time. The chosen caffeine dose $(100 \mathrm{mg}$ ) was slightly higher than the amount of caffeine usually contained in a European cup of coffee (80 mg).

We aimed to study a possible long-term habituation of the MMN by the introduction of four MMN measurements for each condition (one baseline and three consecutive measurements after drug intake). This design also enabled us to investigate possible drug effects over time. Drug effects on more controlled attention processes were additionally assessed by a visual attention task.

\section{METHODS}

\section{Subjects}

A total of 12 young healthy subjects (six males) with an average age of 24.2 years (range from 20 to 27 years) took part in the study. Subjects were not included if they had any history of neurologic, psychiatric, or other medical disease, or if they had first-degree relatives with psychiatric diseases. All subjects were students paid for their participation $(80 \varepsilon$ total). In all, 10 of them were modest coffee consumers (0.52 cups per day), two consumed no coffee at all. None of the participants reported a regular intake of any drug, except contraceptives, but two subjects (both male) were occasional cannabis users. Subjects were instructed to avoid any smoking, alcohol and coffee drinking, or intake of any other drug (except contraceptives) $48 \mathrm{~h}$ prior to a session. All subjects were known from earlier experiments or were tested before their participation whether they produced stable neuromagnetic fields with a sufficient signal-to-noise ratio. After thorough information all participants gave written informed consent. The study was approved by the local ethics committee of the University of Jena.

\section{Procedure}

Subjects were tested on three experimental days which were separated by at least $48 \mathrm{~h}$ in each of the following conditions: after the administration (a) of $1.25 \mathrm{mg}$ lorazepam (Tavor Expidet ${ }^{\circledR}$, Whyeth), (b) of $100 \mathrm{mg}$ caffeine (Coffeinum purum, Berlin-Chemie) and (c) of a placebo pill. All drugs were given with $100 \mathrm{ml}$ water. The order of conditions was counterbalanced and all the sessions took place at the same daytime (starting about 0830). Subjects and the junior investigator (VM) collecting the data were not aware of the drug that was applied. However, since benzodiazepines affect the capability for driving, subjects were told after the lorazepam session that they had been given a benzodiazepine and that they had to avoid car driving or cycling for the next $48 \mathrm{~h}$. If necessary or wished by the subjects, they were afterwards accompanied to their home.

Neuromagnetic fields were recorded within a magnetically shielded room (Vacuumschmelze, Hanau, Germany) about $25 \mathrm{~min}$ before drug intake and 45, 105, $225 \mathrm{~min}$ thereafter. In order to obtain information about the impact of the applied drugs on controlled attention processes, the participants were further required to perform a computer administered attention test (Continuous Performance Test Munich-CPT-M) 60 min before the drug application and $25,85,145$, and $205 \mathrm{~min}$ after it. Blood pressure (BP) and pulse (PR) were measured by an automatic sphygmomanometer (Bosch medicus, Bosch + Sohn, Jungingen, Germany) before drug application, immediately after it and then every $30 \mathrm{~min}$.

\section{CPT}

The CPT-M contains a pseudorandomized sequence of numerals $(2,4,6,8,0)$, with a low background contrast presented at a stimulus frequency of $1 / \mathrm{s}$. In our experiment, each numeral appeared for $42 \mathrm{~ms}$ on the computer screen. Subjects were asked to press the space key as fast as possible when the target stimulus (numeral 0) appeared. Targets had a probability of $p=0.25$. Before testing, subjects were introduced to the CPT-M by learning trials. Overall, the CPT-M is a relatively exclusive attention test with a low load of working memory (Kathmann et al, 1996). Although it contains nearly no memory component, the test is quite difficult to perform because of its high stimulus frequency and the low contrast of the stimuli. Kathmann et al (1996) demonstrated the sensitivity of this test for the study of pharmacological interventions, by investigating the effects of lorazepam, alcohol, and scopolamine in healthy subjects. The reader is referred for more detailed information on the CPT-M to Kathmann et al (1996) and Mass et al (2000).

\section{Neuromagnetic Recordings}

Recordings were carried out with a 31-channel neuromagnetometer system (Philips Medical Systems, Hamburg, Germany) positioned contralaterally to the stimulated ear over the left temporal lobe. The trials had a length of $512 \mathrm{~ms}$ with a $100 \mathrm{~ms}$ prestimulus interval, and a sampling rate of $1000 \mathrm{~Hz}$ was used. The sensor array (diameter about $150 \mathrm{~mm}$ ) was centered over the T3 location of the international 10-20 electrode placement system. Subjects were instructed to ignore the tones and to inhibit any eye movements. The localization of the head before and after each block was provided by means of five orthogonal coils of $5 \mathrm{~mm}$ diameter affixed to the head. Prior to each session, the positions of these coils were digitized in relation to three anatomical landmarks (left and right preauricular points, and nasion) by an Isotrak II system (Polhemus Inc., Colchester, VT, USA). These reference points were employed in order to match the MEG data with high-resolution magnetic resonance images performed on a $1.5 \mathrm{~T}$ GYROSCAN (Philips Medical Systems, Hamburg, Germany; recording parameters: 3D-Flash-sequence producing 256 sagittal images of $1 \mathrm{~mm}$ slice thickness with a $256 \times 256$ matrix covering the entire head). The digitizing of the coil positions was repeated after the second recording and at the end of the sessions in order to control the coil positions over the total experiment. The electrooculogram (EOG) was recorded with a pair of sintered Nihon-Kohden silver-silver 
chloride electrodes, one positioned below and one on the outer canthus of the left eye.

Subjects were stimulated monaurally, lying on their right side on a vacuum cushion. The head was resting on a separate cushion which had an aperture for the right ear. This aperture was connected to a funnel-shaped ending of a plastic tube used for delivering the computer-generated pure tones. At the baseline measurement, the positioning of the biomagnetometer was checked by a test block consisting of 96 auditory stimuli. After a possible correction of the biomagnetometer position, the oddball paradigm was recorded. For all following measurements, the tilting of the biomagnetometer and the position of the bed and vacuum cushions were kept constant in order to reduce the variance of the biomagnetometer position between the measurements of a session.

A $1000 \mathrm{~Hz}$ tone with $50 \mathrm{~ms}$ length at $85 \mathrm{~dB}$ sound pressure level (SPL) served both as stimulus in the test session and as standard tone in the oddball paradigm. Within the oddball paradigm, three types of deviant tones were employed (frequency deviant: $1050 \mathrm{~Hz}$ tone with $50 \mathrm{~ms}$ length at $85 \mathrm{~dB}$; duration deviant: $1000 \mathrm{~Hz}$ tone with $100 \mathrm{~ms}$ length at $85 \mathrm{~dB}$; intensity deviant: $1000 \mathrm{~Hz}$ tone with $50 \mathrm{~ms}$ length at $75 \mathrm{~dB}$ ). The three types of deviants occurred with approximately $10 \%$ pseudorandom probability within the same trial block, consisting of 792 trials. Each deviant was followed by at least one standard tone. The stimulus onset asynchrony (SOA) varied from 800 to $1800 \mathrm{~ms}$ in the test session. In the oddball paradigm, a fixed SOA of $1000 \mathrm{~ms}$ was chosen.

\section{Source Reconstruction}

The first step of data analysis represented the correction of eye artefacts by a regression-based algorithm (Meier et al, 1998). Within this procedure, all trials with a signal power of more than $2.5 \mathrm{pT}$ due to artefacts were singled out automatically. The MMNm was calculated by subtracting the response to standard tones, obtained in the oddball paradigm, from the response to each deviant type. Responses to standard tones following deviants were eliminated. The source reconstruction was performed by means of the software package CURRY 3.0 $0^{\mathrm{TM}}$ (Neuroscan, Sterling, Virginia, USA). Data were digitally bandpass filtered $(1-40 \mathrm{~Hz}$, transition $3 \mathrm{~Hz}$ highpass and $10 \mathrm{~Hz}$ lowpass) and a common average rejection was applied. Exemplary data are shown in Figure 1.

The aim of the study was the comparison of the MMNm and $\mathrm{N} 100 \mathrm{~m}$ strength between different time points (baseline $v s$ later measurements) under different drug conditions. Although we put some emphasis to place the subject under the biomagnetometer in a very similar way, a certain variance in the placement of the subjects could not be avoided. Since the amplitudes of the neuromagnetic fields are influenced by the distance between the subject and the biomagnetometer, we decided to analyze the strength of the reconstructed source (dipole moment) as dependent variable instead of the field power. The calculated N100m and MMNm dipole position was kept constant within a session in order to rule out further an influence of dipole location on the dipole strength between different time points of one session. For this purpose, a singular value decomposition (svd) was calculated for the $\mathrm{N} 100 \mathrm{~m}$ in the time range between 70 and $140 \mathrm{~ms}$ and for the MMNm between 100 and $250 \mathrm{~ms}$. The svd decomposes the averaged signal into mathematically orthogonal signal components. For the dipole reconstruction, the first four signal components were used. In a first step, a source was reconstructed at the peak of the first signal component for all four measurements (baseline, 45, 105, $225 \mathrm{~min}$ ) of a session. A single moving equivalent dipole embedded in a spherical volume conductor was used as source model. This first step was performed for the $\mathrm{N} 100 \mathrm{~m}$ and for the MMNm of each kind deviant separately. In a second step, the obtained dipole position was affixed and the dipole strength (qb) was

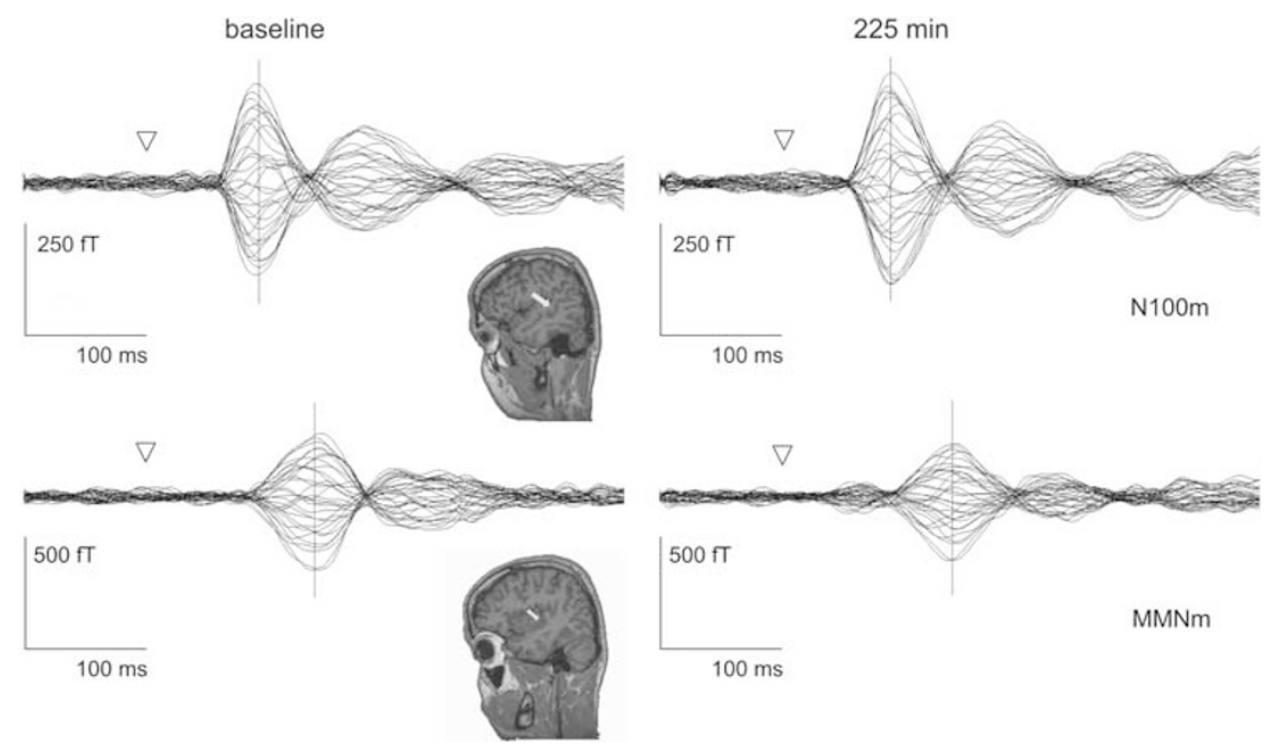

Figure I Individual data at baseline (left column) and at $225 \mathrm{~min}$ (right column) on the NI00m (top) and MMNm on frequency deviants (bottom) in the placebo condition; the stimulus onset is indicated by the downwards tilted apex, the peak latency of the NI00m and MMNm are marked by the dotted vertical line; the reconstructed source of both components are depicted as a white arrow projected on sagittal MR slices; the NI00m was quite stable, while a decrease of the MMNm was observed during the sessions even in the placebo condition. 
calculated at the peak maximum of the first signal component $\left(t_{\max }\right)$ for each individual measurement. The dipole position of the MMNm of different kind of deviants (frequency, duration, and intensity deviants) and of different sessions were, however, allowed to vary. The introduction of four measurements into the source reconstruction was not thought to give the most reliable dipole position, but to represent the best estimator for the evoked brain activity in all four individual measurements. A goodness-of-fit criterion was not introduced since both the application of lorazepam and measurement repetition were expected to diminish the signal-to-noise ratio and a diminished signal-to-noise ratio leads to a decreased goodness-of-fit. However, a dipole solution was only accepted if the dipole was located in the temporal gyrus at least $25 \mathrm{~mm}$ apart from the sagittal midline and if it was orientated in an inferior-posterior direction.

The dipole data were projected into a head-based Cartesian system of coordinates and normalized to the individual head size, in order to obtain interindividually comparable coordinates. The origin of this coordinate system was set into the commissura anterior, projected on a plane through the fissura longitudinalis. The $y$-axis runs through the commissura posterior. The $z$-axis is tilted toward the top of the head (Rosburg et al, 2001).

\section{Statistics}

All original baseline measurements were compared between DRUG conditions (caffeine $v s$ benzodiazepine $v s$ placebo). Data were then normalized by subtraction of the baseline measurement values from the measurements taken during the corresponding session (ie by a linear transformation). Standard deviations remained unchanged. Subsequently, the normalized values were compared by means of repeated measure analyses of variance (ANOVA). The normalization avoids the influence of slight (mostly nonsignificant) differences in the baseline measurements on the comparison between conditions after drug intake. Except the baseline comparison, all statistics were performed with normalized data. In figures and tables, the original (not transformed) data are presented.

Variables obtained in the CPT measurement, namely the signal detection performance $\left(d^{\prime}\right)$, the natural-logarithmic response bias $(\beta)$ (Velden 1982), and reaction time $(\mathrm{rt})$, were analyzed by means of repeated measure ANOVA. DRUG and TIME (25 vs 85 vs 145 vs 205 min after drug exposure) served as factors for repeated measurements in order to evaluate whether DRUG exposure had a general influence on test performance and/or whether the impact of DRUG depended on the analyzed TIME point.

The neuromagnetic data were also analyzed by ANOVA. Differences in dipole location between DRUG conditions were assessed for both $\mathrm{N} 100 \mathrm{~m}$ and MMNm data. Since only one minor difference in dipole location between DRUG conditions was detected, results on these comparisons are not presented. As for other data, possible differences between the baseline measurements of each condition in latency and dipole moment were assessed. Effects of DRUG and TIME (45 vs 105 vs $225 \mathrm{~min}$ after drug exposure) on normalized latency and dipole moment were analyzed in a repeated measure ANOVA. For the MMNm data, the kind of
TYPE of DEVIANCE was treated also as a factor of repeated measurement (frequency $v s$ duration $v s$ intensity deviance). Possible effects were further evaluated by ANOVAs for each condition or each time point separately.

DRUG and TIME (5 vs 35 vs 65 vs 95 vs 125 vs 155 vs 185 vs 215 vs $245 \mathrm{~min}$ after drug exposure) served as factors for the repeated measurement in order to evaluate effects of DRUG exposure on the vegetative system. All significant DRUG effects were followed by a pairwise comparison. Statistical tests were computed with the SPSS software package 11.5 (SPSS Inc., Chicago, IL, USA). Where necessary, a Greenhouse-Geisser correction was performed and significant $\varepsilon$-values are reported for all comparisons, resulting in $p$-values of $<0.05$.

\section{RESULTS}

\section{CPT}

All CPTs were performed successfully except for the final testing $(205 \mathrm{~min})$ in the lorazepam condition of one subject. In that testing, the subject was not able to follow the test instructions any more and pressed the response key unsystematically, resulting in an exorbitant high error rate. The data were replaced by the data set of the testing before ( $145 \mathrm{~min})$ in order to include the total data set into the final analyses.

CPT performance at baseline did not differ between the three DRUG conditions $\left(d^{\prime}: \mathrm{F}_{2,22}=0.185, \quad \mathrm{NS} ; \quad \beta\right.$ : $\mathrm{F}_{2,22}=0.132, \mathrm{NS} ; \mathrm{rt}: \mathrm{F}_{2,22}=0.843$, NS), indicating that the performance at the beginning of the experiment was in all conditions at a similar level. The administration of lorazepam resulted in a reduced signal detection performance $d^{\prime}$, both compared to the placebo and caffeine condition (DRUG: $\mathrm{F}_{2,22}=10.028, \quad p<0.01, \quad \varepsilon=0.673$; DRUG $\times$ TIME: $F_{6,66}=3.365, p<0.01$ ) (Figure 2). It did not differ between the placebo and caffeine condition. The

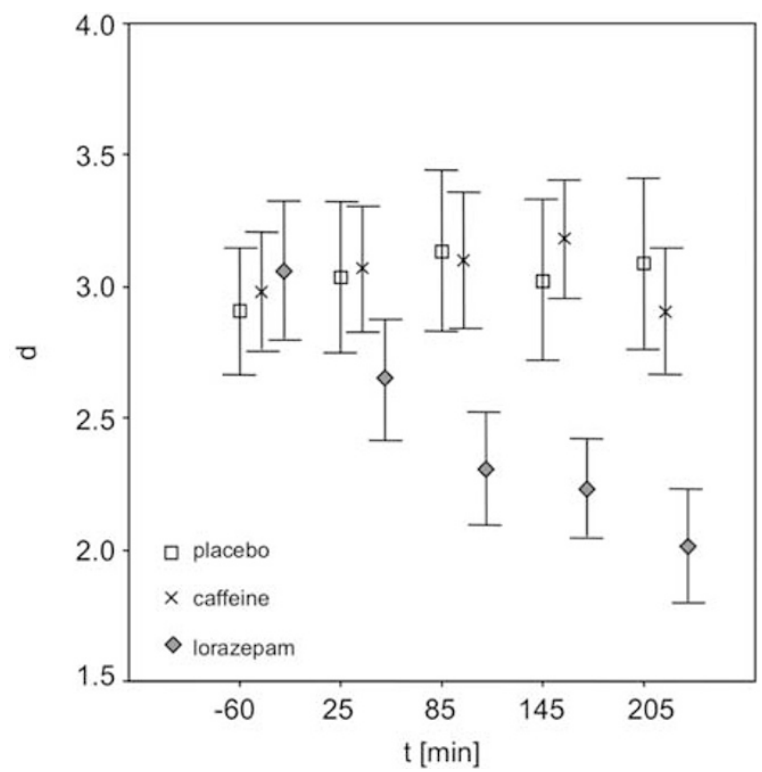

Figure 2 Mean CPT signal detection performance $d^{\prime \prime}$ ( \pm standard error $\mathrm{SE})$ for the three conditions (placebo, caffeine, lorazepam) and five time points (baseline, 25, 85, 145, 205 min after drug application). 
response bias $\beta$ was not affected by DRUG $\left(\mathrm{F}_{2,22}=1.322\right.$, $\mathrm{NS})$ or by a DRUG $\times$ TIME interaction $\left(\mathrm{F}_{6,66}=0.426, \mathrm{NS}\right)$. The slightly accelerated reaction times in the caffeine condition failed to reach significance (DRUG: $\mathrm{F}_{2,22}=3.264, \mathrm{NS}$ ).

Since the influence of DRUG on $d^{\prime}$ is modulated by TIME, possible training effects on CPT performance or systematic alterations within one experimental day can be analyzed only within one condition. The administration of placebo $\left(\mathrm{F}_{4,44}=0.595, \mathrm{NS}\right)$ and caffeine $\left(\mathrm{F}_{4,44}=0.991, \mathrm{NS}\right)$ had no impact on $d^{\prime}$. Prominent alterations of $d^{\prime}$ were observed under lorazepam. Within the lorazepam session, $d^{\prime}$ decreased significantly $\left(\mathrm{F}_{4,44}=12.866, p<0.001\right)$, with a significant reduction after 25 min compared to baseline and a continuing decrease over the total experiment (Figure 2). The administration of lorazepam had, as expected from the initial ANOVA, no influence on the reaction time $\left(\mathrm{F}_{4,44}=0.726, \mathrm{NS}\right)$ and response bias $\beta\left(\mathrm{F}_{4,44}=0.748, \mathrm{NS}\right)$.

\section{N100m}

The $\mathrm{N} 100 \mathrm{~m}$ data could be analyzed in all subjects and conditions. At baseline, the comparison of $\mathrm{N} 100 \mathrm{~m}$ latency and dipole moment revealed no significant differences between the conditions $\left(F_{2,22}=0.207, N S ; F_{2,22}=1.667\right.$, NS, respectively). In the repeated measure ANOVA (factors DRUG and TIME), a significant influence of DRUG on the N100m dipole moment $\left(\mathrm{F}_{2,22}=7.820, p<0.01\right)$, a significant influence of TIME on $\mathrm{N} 100 \mathrm{~m}$ latency $\left(\mathrm{F}_{2,22}=7.353\right.$, $p<0.01$ ), and a significant DRUG $\times$ TIME interaction for the $\mathrm{N} 100 \mathrm{~m}$ dipole moment $\left(\mathrm{F}_{4,44}=2.687, p<0.05\right)$ were detected. A DRUG $\times$ TIME interaction for the $\mathrm{N} 100 \mathrm{~m}$ latency failed to reach significance after GreenhouseGeisser correction $\left(\mathrm{F}_{4,44}=2.851\right.$, NS, $\left.\varepsilon=0.556\right)$.

Owing to the DRUG $\times$ TIME interactions, a separate analysis of effects for each condition and time point was performed. Descriptive data are given in Tables 1 and 2.
Within the caffeine condition, the $\mathrm{N} 100 \mathrm{~m}$ dipole moment and its latency remained stable during the experimental session $\left(\mathrm{F}_{3,33}=0.057, \mathrm{NS} ; \mathrm{F}_{3,33}=0.641\right.$, NS, respectively). In the placebo condition, the dipole moment also remained stable $\left(\mathrm{F}_{3,33}=0.457, \mathrm{NS}\right)$, but the $\mathrm{N} 100 \mathrm{~m}$ latency increased during the session $\left(\mathrm{F}_{3,33}=5.416, p<0.01\right)$. In the benzodiazepine condition, the dipole moment decreased $\left(\mathrm{F}_{3,33}=5.920, p<0.05, \varepsilon=0.499\right)$ and the $\mathrm{N} 100 \mathrm{~m}$ latency increased $\left(\mathrm{F}_{3,33}=15.253, p<0.001\right)$. The $\mathrm{N} 100 \mathrm{~m}$ dipole moment in the benzodiazepine condition was significantly smaller than in the other two condition at $105 \mathrm{~min}$ $\left(\mathrm{F}_{2,22}=11.547, p<0.001\right)$ and $225 \mathrm{~min} \quad\left(\mathrm{~F}_{2,22}=4.473\right.$, $p<0.05)$. The mean $\mathrm{N} 100 \mathrm{~m}$ dipole moments for each condition and time point are shown in Figure 3. Latency differences between conditions did not reach significance.

\section{MMN}

The MMNm data could be analyzed in all subjects and conditions except for the MMNm of intensity deviants in the benzodiazepine condition in one subject. In this particular case, no MMNm was observable in the baseline measurement, as well as in later measurements $(45,105$, $225 \mathrm{~min}$ ). Therefore, this individual $\mathrm{MMNm}$ data set of intensity deviants was excluded from analysis and the number of included cases was reduced from 12 to 11 for some analyses. At baseline, the MMNm dipole moment and latencies of all three deviants did not differ significantly between conditions (dipole moment: frequency deviants: $\mathrm{F}_{2,22}=0.093$, NS; duration deviants: $\mathrm{F}_{2,22}=2.330$, NS; intensity deviants: $F_{2,20}=1.200, N S$; latency: frequency deviants: $\quad \mathrm{F}_{2,22}=1.050, \quad \mathrm{NS}$; duration deviants: $\mathrm{F}_{2,22}=2.653$, NS; intensity deviants: $\mathrm{F}_{2,20}=0.473$, NS).

In the repeated measure ANOVA (factors TYPE of DEVIANCE, DRUG, and TIME), a significant DRUG effect was detected for the dipole moment of the MMNm $\left(\mathrm{F}_{2,22}=5.334, p<0.05\right)$, with lower dipole moments in the

Table I The Dipole Moment of the NI00m and MMNm in the Three Conditions (P — placebo, C-caffeine, B-benzodiazepine) and at the Four Time Points of Measurement (25 min before Drug Intake and 45, 105, 225 min after It)

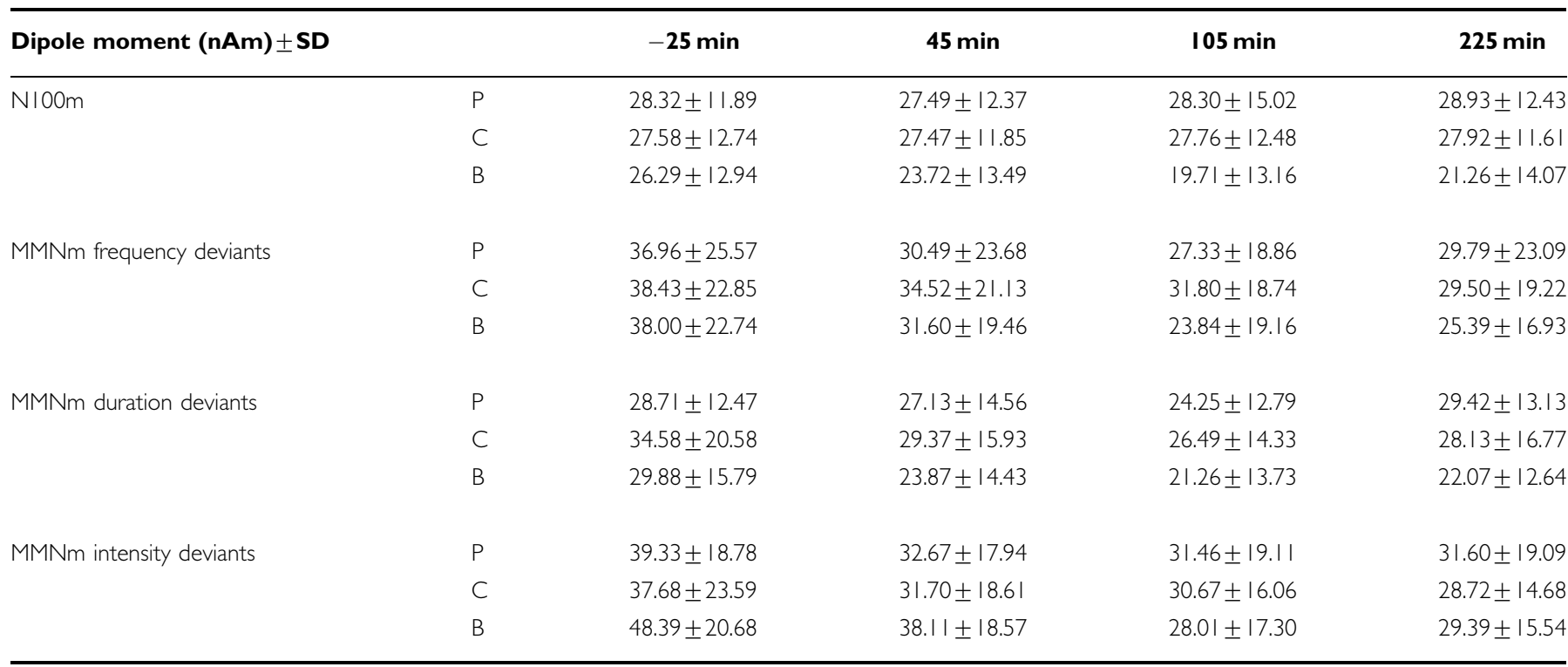


Table 2 According to Table I, the Peak Latency of the NI00m and MMNm in the Three Conditions and at the Four Time Points of Measurement

\begin{tabular}{|c|c|c|c|c|c|}
\hline Latency $(\mathbf{m s}) \pm S D$ & & $-25 \mathrm{~min}$ & $45 \mathrm{~min}$ & $105 \mathrm{~min}$ & $225 \mathrm{~min}$ \\
\hline \multirow[t]{3}{*}{$\mathrm{NIOOm}$} & $P$ & $101.4 \pm 10.3$ & $103.5 \pm 9.9$ & $103.4 \pm 8.6$ & $106.6 \pm 10.4$ \\
\hline & C & $100.8 \pm 8.7$ & $99.6 \pm 9.2$ & $101.1 \pm 8.2$ & $101.7 \pm 8.7$ \\
\hline & $\mathrm{B}$ & $102.2 \pm 8.1$ & $105.2 \pm 7.8$ & $110.3 \pm 9.8$ & $108.6 \pm 9.2$ \\
\hline MMNm frequency deviants & $P$ & $159.7 \pm 14.9$ & $161.3 \pm 19.6$ & $161.1 \pm 15.3$ & $160.1 \pm 20.7$ \\
\hline \multirow[t]{3}{*}{ MMNm duration deviants } & $P$ & $166.0 \pm 7.4$ & $172.2 \pm 13.2$ & $171.4 \pm 12.0$ & $173.5 \pm 10.6$ \\
\hline & C & $173.2 \pm 14.6$ & $173.3 \pm 17.1$ & $180.3 \pm 19.4$ & $173.3 \pm 10.9$ \\
\hline & B & $174.4 \pm 11.9$ & $171.6 \pm 13.0$ & $170.6 \pm 13.0$ & $170.1 \pm 14.8$ \\
\hline
\end{tabular}

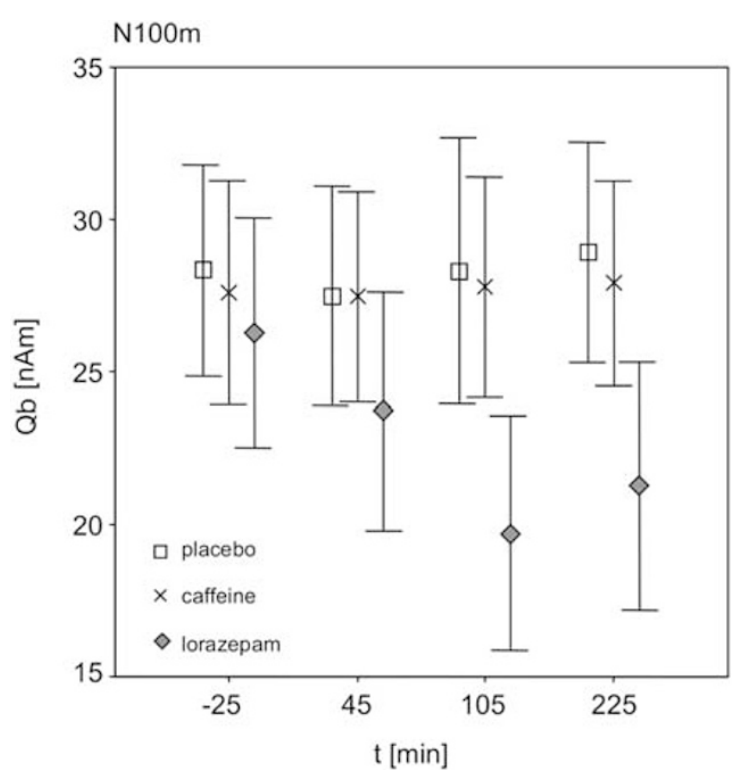

Figure 3 Mean NI00m dipole moment $( \pm \mathrm{SE})$ for the three conditions and four time points (baseline, 45, 105, $225 \mathrm{~min}$ after drug application).

benzodiazepine condition than in the two other condition. Besides that, a highly significant effect of TIME $\left(\mathrm{F}_{2,22}=10.169, p \leqslant 0.001\right)$ and a significant interaction between TIME and DRUG $\left(\mathrm{F}_{4,44}=2.812, p<0.05\right)$ could be revealed for the MMNm dipole moment.

Owing to the significant TIME`DRUG interaction for the MMNm dipole moment, the influence of DRUG was analyzed for each time point separately and the influence of TIME for each DRUG condition. Significant differences between DRUG conditions were found at $105 \mathrm{~min}$ $\left(\mathrm{F}_{2,22}=7.579, \quad p<0.01\right)$ and $225 \mathrm{~min} \quad\left(\mathrm{~F}_{2,22}=8.156\right.$, $p<0.01, \varepsilon=0.664)$, with lower dipole moments in the benzodiazepine condition than in the two other conditions. A decrease of the MMNm dipole moment over TIME was

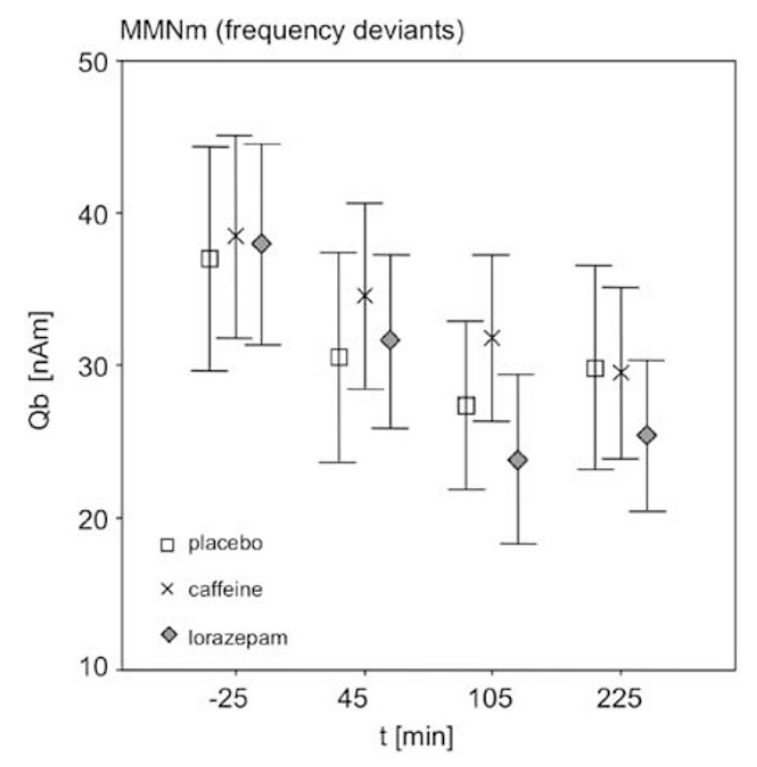

Figure 4 Exemplarily for the $M M N m$, the mean dipole moment of the $\mathrm{MMNm}$ on frequency deviants $( \pm \mathrm{SE})$ for the three conditions and four time points.

observed for all conditions, with the strongest decrease over TIME in the benzodiazepine condition (placebo: $\mathrm{F}_{3,33}=9.477, p<0.001, \varepsilon=0.714$; caffeine: $\mathrm{F}_{3,33}=8.710$, $p<0.01, \quad \varepsilon=0.554 ; \quad$ benzodiazepine: $\quad \mathrm{F}_{3,33}=29.984$, $p<0.001)$. Exemplary data on MMNm dipole moment are shown in Figure 4. Descriptive data are given in Table 1.

For the latency of the MMNm, main factors alone had no significant influence on the data, but a significant interaction between TYPE of DEVIANCE and DRUG was obtained $\left(\mathrm{F}_{4,44}=4.688, p<0.01\right)$. Owing to this interaction, the DRUG effects on the MMNm latency were analyzed for each kind of deviant. Within the placebo condition, a significant increase of the MMNm latency of duration deviants was observable $\left(\mathrm{F}_{3,33}=3.039, p<0.05\right)$, which 
seems to be caused by a decreased latency at baseline, in comparison to the other two conditions, and will, therefore, not be interpreted. However, a significant effect was detected for the $\mathrm{MMNm}$ latency of intensity deviants $\left(\mathrm{F}_{2,20}=6.626, p<0.05, \varepsilon=0.664\right)$, with significant longer latencies in the benzodiazepine compared to the caffeine condition. Compared to the baseline, the latency was significantly increased after the administration of benzodiazepines at $105\left(\mathrm{~F}_{1,10}=12.941, p<0.01\right)$ and $225 \mathrm{~min}$ $\left(\mathrm{F}_{1,10}=4.994, p<0.05\right)$. The MMNm latency of other kinds of deviants was not affected by the intake of benzodiazepine (frequency deviants: $\mathrm{F}_{3,33}=0.646$, NS; duration deviants: $\left.\mathrm{F}_{3,33}=0.406, \mathrm{NS}\right)$. Descriptive data are given in Table 2 .

\section{Blood Pressure and Pulse}

These data were obtained mainly for the safety of the participants. All blood pressure and pulse values but for one person at one sample point were obtained successfully. The missing values were replaced by the values of the previous measurement.

The average BP values at baseline did not differ significantly between conditions (systolic $\mathrm{BP}: \mathrm{F}_{2,22}=0.150$, NS; diastolic BP: $F_{2,22}=2.778$, NS). In the subsequent ANOVA with the factors DRUG and TIME, DRUG effects were revealed for both normalized BP values (systolic BP: $\mathrm{F}_{2,22}=6.418, \quad p<0.01 ; \quad$ diastolic $\mathrm{BP}: \mathrm{F}_{2,22}=10.393$, $p \leqslant 0.001)$, with larger values in the caffeine condition compared to the two other conditions. Compared to baseline, the effects of caffeine were observable after $35 \mathrm{~min}$.

The average PR did not differ between conditions at baseline $\left(\mathrm{F}_{2,22}=1.736\right.$, NS). After drug administration differences were observed between conditions and sample points (DRUG: $\mathrm{F}_{2,22}=2.700$, NS; TIME: $\mathrm{F}_{8,88}=3.535$; $p \leqslant 0.001 ; \quad$ DRUG $\times$ TIME: $\left.F_{16,176}=2.369, p<0.01\right)$. The TIME effects were partly due to a decrease from baseline to $5 \mathrm{~min}$ after drug exposure in all three conditions. As shown in ANOVAs for each condition separately, there were significant increases in $\mathrm{PR}$ after drug exposure in the caffeine $\left(\mathrm{F}_{7,77}=3.327, p<0.01\right)$ and lorazepam condition $\left(\mathrm{F}_{7,77}=3.350, p<0.01\right)$, but not in the placebo condition $\left(\mathrm{F}_{7,77}=0.221, \mathrm{NS}\right)$.

\section{DISCUSSION}

The main results of our study may be summarized as follows: lorazepam had a strong impact on CPT performance: a decrease in the signal detection performance $d^{\prime}$ was observed, starting $25 \mathrm{~min}$ after the intake of lorazepam and continuing until the end of the experiment. After lorazepam, the $\mathrm{N} 100 \mathrm{~m}$ was decreased in its dipole moment, compared to the other two conditions. The decrease in the N100m dipole moment became statistically evident in the second measurement after drug intake (105 min). In all conditions, the MMNm dipole moment decreased in the course of a session. This decrease was enlarged after lorazepam intake. Only for the MMNm of intensity deviants, a significant increase in latency was observed after lorazepam intake. Lorazepam and caffeine led to an increase of pulse rate, compared to placebo. Caffeine had no significant effects on behavioral measures and neuromag- netic recordings. The findings will be discussed now in more detail.

\section{CPT Performance}

An impact of lorazepam on CPT performance has been expected from known effects of lorazepam, as well as from earlier studies (Duka et al, 1995; Fluck et al, 2001; Kathmann et al, 1996; Krystal et al, 1998; Mass et al, 2000; Micallef et al, 2001; Post et al, 1997). The data illustrate that the performance in controlled visual attention was clearly impaired by lorazepam intake. This impairment is not totally self-evident, since for example Fluck et al (2001) found significant effects on attention after $2.5 \mathrm{mg}$, but not after $1 \mathrm{mg}$ lorazepam, as assessed by a digit cancellation and digit symbol substitution test. A higher lorazepam dose was deliberately not applied in the current study since it would have increased the likelihood that subjects would fall asleep during the MEG measurements. Without the simultaneous examination of controlled visual attention processes, one would be unable to interpret the significance of any drug effects on electrophysiological parameters, especially in the study of MMN where no responses are required. A slowing of reaction time by lorazepam, as described earlier by Post et al (1997) and Stephenson et al (2003), was not observed.

\section{Neuromagnetic Recordings}

After intake of placebo, the $\mathrm{N} 100 \mathrm{~m}$ dipole moment remained quite stable, while the latency of the $\mathrm{N} 100 \mathrm{~m}$ increased slightly. Since the latency increase and the amplitude decrease of the $\mathrm{N} 100 \mathrm{~m}$ seem to represent two independent processes of habituation (Rosburg et al, 2002), the sole $\mathrm{N} 100 \mathrm{~m}$ latency increase without an amplitude/dipole moment decrease is not unexpected. The stable $\mathrm{N} 100 \mathrm{~m}$ dipole moment within the placebo and caffeine condition indicates that subjects were equally attentive during the session, as attention was found to affect the N100m amplitude (Hari et al, 1989; Woldorff et al, 1993). Therefore, the strong TIME effect on the MMNm dipole moment, observed in all conditions and for all kinds of deviants, cannot be explained by attention effects.

Recent findings on MMN habituation (May et al, 1994; Baldeweg et al, 1999, McGee et al, 2001) are, on the first glance, somewhat contradicting. However, for the interpretation of these results one has to consider the extremely diverging duration of recording times. Taken together with those recent studies, our findings suggest that a habituation of the MMN occurs in the range of minutes. As reason for the observed habituation of the MMN, we assume that the repeating acoustic deviance itself forms a memory trace, since it was shown that tone repetition can also elicit an MMN in the context of permanently varying stimulation (Horvath et al, 2001; Wolff and Schröger, 2001; Rosburg, 2004). The memory trace of the acoustic deviance might be strengthened over time, as the kind, occurrence, and likelihood of deviance are somewhat predictable. Subjects get used to a permanent deviation and from an ecological viewpoint a permanent deviation 'deserves' less attention. 
Caffeine had no significant effects on neuromagnetic recordings. An increase of $\mathrm{N} 100 \mathrm{~m}$ latency was not observed over time after caffeine, but latency differences between the placebo and caffeine condition did not reach significance. Effects of caffeine on event-related components might become observable only at higher doses. Similar to our current finding, Hirvonen et al (2000) observed no alteration of the MMN and other event-related components after $100 \mathrm{mg}$ caffeine. After intake of $500 \mathrm{mg}$ caffeine, Kawamura et al (1996) observed an increase of P300 amplitude (for comparison: $500 \mathrm{mg}$ caffeine would be equivalent to six European cups of coffee). Dutch researchers revealed an increase of N100, N200b, and P300 amplitudes after administration of $250 \mathrm{mg}$ caffeine (Lorist et al, 1995; Ruijter et al, 2000a, b, c).

The lorazepam intake had, in contrast to caffeine, very pronounced effects on neuromagnetic fields. It led to a decrease of the $\mathrm{N} 100 \mathrm{~m}$ dipole moment by about $20-25 \%$, becoming evident in the second measurement after the drug intake (105 $\mathrm{min})$. This decrease could be easily detected since the $\mathrm{N} 100 \mathrm{~m}$ remained relatively stable over time in the control conditions. The finding of a $\mathrm{N} 100 \mathrm{~m}$ dipole moment decrease by lorazepam is well in line with earlier EEG (Rockstroh et al, 1991; Semlitsch et al, 1995) and MEG (Sinton et al, 1986) studies, reporting an N100/N100m amplitude decrease by benzodiazepine intake. Interestingly, no N100 amplitude reduction was observed in the study by Nakagome et al (1998), who revealed a reduction of the $M M N$ as an overnight effect of triazolam intake.

As outlined, the MMNm dipole moment decreased within a recording session in all conditions. Its decrease amounted to about $20-25 \%$ on average already in the control conditions. Lorazepam intake further aggravated the decrease of the MMNm dipole moment over time. The current finding is in contrast to other studies, showing no major effects of benzodiazepines (Murakami et al, 2002) and anxiolytics/hypnotics, including benzodiazepines ( $\mathrm{Ka}-$ sai et al, 2002) on MMN amplitude, and in line with the study of Nakagome et al (1998). Our finding emphasizes the necessity to take the usage of benzodiazepines into account for clinical investigations of the MMN.

Summing up the current and earlier reported effects of benzodiazepines on event-related potentials (ERPs), midlatency ERPs (including P50) seem to be unaffected by benzodiazepines (Schwender et al, 1993), while the amplitudes of most if not all long-latency ERPs are decreased (Milligan et al, 1989; Nichols and Martin, 1993; Rockstroh et al, 1991; Semlitsch et al, 1995). Besides an enforced inhibition of neural circuits involved in the MMN generation by GABAergic interneurons, the observed reduction of the MMN could also stem from an attenuated efficacy in sensory information processing, as reflected by the decreased $\mathrm{N} 100 \mathrm{~m}$ (as a bottom-up process). Conversely, as the $\mathrm{N} 100 \mathrm{~m}$ is known to be modulated by controlled attention (Hari et al, 1989; Woldorff et al, 1993), the decreased $\mathrm{N} 100 \mathrm{~m}$ could result also from hampered attention functions (as a top-down process). An indirect modulation of other neurotransmitters as a reason for the reduced MMN also has to be considered, although an influence of a downregulated dopaminergic transmission can be regarded as unlikely since the direct application of the $D_{2}$ receptor antagonist haloperidol was found to have no effect on the MMN amplitude (Kähkönen et al, 2001, 2002; Pekkonen et al, 2002).

The latency of the MMNm was less affected by drug condition. Only for the MMNm of intensity deviants, a significant increase in latency was observed after lorazepam intake. It is difficult to say why only the processing of intensity deviance was slowed down by lorazepam. A differential impact of scopolamine exposure was reported for the MMN of frequency and duration deviants (Pekkonen et al, 2001). Also, the deficit in MMN generation in schizophrenia was more pronounced for a deviance in tone duration than in frequency (Michie et al, 2000). Therefore, a differential impact of drug exposure on the MMN of different kind of deviants is not necessarily unexpected. The current finding of a delayed MMN of intensity deviants might possibly be explained by the fact that those deviants were reduced in intensity and, therefore, in the state of decreased vigilance less attention attracting. Further research is required here.

The decrease of MMNm dipole moment after lorazepam seems not to represent the sum of habituation effects as observed in the MMNm control conditions and of the benzodiazepine effect as observed in the N100m data, since it amounted to about $30-35 \%$ on average. One reason for this nonadditivity could be that the habituation of the MMN is modulated by intrinsic GABAergic action, that is, an active inhibition process occurs as a result of repeated deviance. In that case, GABAergic inhibitory neurons would be activated already intrinsically and the GABA agonist lorazepam could not unfold its full efficacy. A GABAergic inhibitory mechanism for 'repetition-adaptivity' was very recently proposed by Stephenson et al (2003). Another possible explanation could be that the MMNm generation is less modulated by GABAergic transmission than the $\mathrm{N} 100 \mathrm{~m}$. To clarify this issue, it would be helpful to investigate whether a benzodiazepine antagonist as flumazenil could prevent the MMN habituation. The study of Smolnik et al (1998) contained, unfortunately, only a single measurement after drug administration and cannot resolve this question.

\section{Pulse Rate}

The increase of pulse rate in the lorazepam condition was on the first glance surprising, since no effects on the vegetative system had been expected. An observation similar to ours was also reported by Agelink et al (2002) and Krystal et al (1998). However, a double-blind, randomized, crossover designed study on noninvasive indices of autonomic regulation revealed no time-dependent change of heart rate and no effect on blood pressure and on respiratory frequency within a period of $4 \mathrm{~h}$ after oral administration of $2 \mathrm{mg}$ lorazepam, indicating no considerable effect of lorazepam on sympathetic and parasympathetic processes (Tulen and Man in't Veld 1998). Accordingly, we assume that the observed increase in pulse rate after lorazepam could represent an autonomous or volitional counter-regulation of the lorazepam effects rather than a direct result of the pharmacological profile. 


\section{Conclusion}

Besides the already known effects of benzodiazepines on controlled attention functions, as shown here by a reduced signal detection performance in the CPT-M and possibly also in the reduced $\mathrm{N} 100 \mathrm{~m}$, the ability to detect discontinuities in acoustic input preattentively is hampered by acute benzodiazepine intake. In order to differentiate between direct and indirect drug modulation of the auditory cortex function, studies employing more than one active drug at one time are warranted. The strong habituation of the MMNm emphasizes the importance to keep the recording conditions and especially the recording time in the investigation of clinical groups constant or, as a practical alternative, to introduce recording time as a covariable.

\section{ACKNOWLEDGEMENTS}

We would like to thank Dr Ilonka Kreitschmann-Andermahr and three anonymous reviewers for their helpful comments. The article contains larger parts of the doctoral thesis of Ms Varvara Marinou.

\section{REFERENCES}

Agelink MW, Majewski TB, Andrich J, Mueck-Weymann M (2002). Short-term effects of intravenous benzodiazepines on autonomic neurocardiac regulation in humans: a comparison between midazolam, diazepam, and lorazepam. Crit Care Med 30: 997-1006.

Ahveninen J, Escera C, Polo MD, Grau C, Jääskeläinen IP (2000). Acute and chronic effects of alcohol on preattentive auditory processing as reflected by mismatch negativity. Audiol Neurootol 5: 303-311.

Ahveninen J, Kähkönen S, Pennanen S, Liesivuori J, Ilmoniemi RJ, Jääskeläinen IP (2002). Tryptophan depletion effects on EEG and MEG responses suggest serotonergic modulation of auditory involuntary attention in humans. Neuroimage 16: 1052-1061.

Alain C, Woods DL (1997). Attention modulates auditory pattern memory as indexed by event-related brain potentials. Psychophysiology 34: 534-546.

Baldeweg T, Williams JD, Gruzelier JH (1999). Differential changes in frontal and sub-temporal components of mismatch negativity. Int J Psychophysiol 33: 143-148.

Catts SV, Shelley AM, Ward PB, Liebert B, McConaghy N, Andrews $S$ et al (1995). Brain potential evidence for an auditory sensory memory deficit in schizophrenia. Am J Psychiatry 152: 213-219.

Duka T, Redemann B, Voet B (1995). Scopolamine and lorazepam exert different patterns of effects in a test battery assessing stages of information processing. Psychopharmacology (Berl) 119: 315-324.

Fluck E, Fernandes C, File SE (2001). Are lorazepam-induced deficits in attention similar to those resulting from aging? J Clin Psychopharmacol 21: 126-130.

Hari R, Hämäläinen M, Kaukoranta E, Mäkelä J, Joutsiniemi SL, Tiihonen J (1989). Selective listening modifies activity of the human auditory cortex. Exp Brain Res 74: 463-470.

Hirvonen J, Jääskeläinen IP, Näätänen R, Sillanaukee P (2000). Adenosine A1/A2a receptors mediate suppression of mismatch negativity by ethanol in humans. Neurosci Lett 278: 57-60.

Horvath J, Czigler I, Sussman E, Winkler I (2001). Simultaneously active pre-attentive representations of local and global rules for sound sequences in the human brain. Brain Res Cogn Brain Res 12: $131-144$.
Javitt DC, Doneshka P, Grochowski S, Ritter W (1995). Impaired mismatch negativity generation reflects widespread dysfunction of working memory in schizophrenia. Arch Gen Psychiatry 52: $550-558$.

Kähkönen S, Ahveninen J, Jääskeläinen IP, Kaakkola S, Näätänen $\mathrm{R}$, Huttunen J et al (2001). Effects of haloperidol on selective attention: a combined whole-head MEG and high-resolution EEG study. Neuropsychopharmacology 25: 498-504.

Kähkönen S, Ahveninen J, Pekkonen E, Kaakkola S, Huttunen J, Ilmoniemi RJ et al (2002). Dopamine modulates involuntary attention shifting and reorienting: an electromagnetic study. Clin Neurophysiol 113: 1894-1902.

Kasai K, Yamada H, Kamio S, Nakagome K, Iwanami A, Fukuda M et al (2002). Do high or low doses of anxiolytics and hypnotics affect mismatch negativity in schizophrenic subjects? An EEG and MEG study. Clin Neurophysiol 113: 141-150.

Kathmann N, Wagner M, Satzger W, Engel RR (1996). Vigilanzmessung auf Verhaltensebene: Der Continuous Performance Test München (CPT-M). In: Möller HJ, Engel RR, Hoff P (eds). Befunderhebung in der Psychiatrie. Springer: Wien, New York. pp 331-338.

Kawamura N, Maeda H, Nakamura J, Morita K, Nakazawa Y (1996). Effects of caffeine on event-related potentials: comparison of oddball with single-tone paradigms. Psychiatry Clin Neurosci 50: 217-221.

Kreitschmann-Andermahr I, Rosburg T, Demme U, Gaser E, Nowak H, Sauer H (2001). Effect of ketamine on the neuromagnetic mismatch field in healthy humans. Brain Res Cogn Brain Res 12: 109-116.

Krystal JH, Karper LP, Bennett A, D’Souza DC, Abi-Dargham A, Morrissey $\mathrm{K}$ et al (1998). Interactive effects of subanesthetic ketamine and subhypnotic lorazepam in humans. Psychopharmacology (Berl) 135: 213-229.

Kujala T, Näätänen R (2001). The mismatch negativity in evaluating central auditory dysfunction in dyslexia. Neurosci Biobehav Rev 25: 535-543.

Lorist MM, Snel J, Mulder G, Kok A (1995). Aging, caffeine, and information processing: an event-related potential analysis. Electroencephalogr Clin Neurophysiol 96: 453-467.

Mass R, Wolf K, Wagner M, Haasen C (2000). Differential sustained attention/vigilance changes over time in schizophrenics and controls during a degraded stimulus Continuous Performance Test. Eur Arch Psychiatry Clin Neurosci 250: 24-30.

May P, Tiitinen H, Sinkkonen J, Näätänen R (1994). Long-term stimulation attenuates the transient $40-\mathrm{Hz}$ response. Neuroreport 5: 1918-1920.

McGee TJ, King C, Tremblay K, Nicol TG, Cunningham J, Kraus N (2001). Long-term habituation of the speech-elicited mismatch negativity. Psychophysiology 38: 653-658.

Meier T, Rosburg T, Arnold M, Kreitschmann-Andermahr I, Sauer $\mathrm{H}$, Nowak H et al (1998). Quantification and rejection of ocular artifacts in auditory evoked fields in schizophrenics. Electroencephalogr Clin Neurophysiol 108: 526-535.

Micallef J, Soubrouillard C, Guet F, Le-Guern ME, Alquier C, Bruguerolle B et al (2001). A double blind parallel group placebo controlled comparison of sedative and amnesic effects of etifoxine and lorazepam in healthy subjects. Fundam Clin Pharmacol 15: 209-216.

Michie PT, Budd TW, Todd J, Rock D, Wichmann H, Box J et al (2000). Duration and frequency mismatch negativity in schizophrenia. Clin Neurophysiol 111: 1054-1065.

Milligan KR, Lumsden J, Howard RC, Howe JP, Dundee JW (1989). Use of auditory evoked responses as a measure of recovery from benzodiazepine sedation. J Roy Soc Med 82: 595-597.

Murakami T, Nakagom K, Kamio S, Kasai K, Iwanami A, Hiramatsu $\mathrm{K}$ et al (2002). The effects of benzodiazepines on event-related potential indices of automatic and controlled 
processing in schizophrenia: a preliminary report. Prog Neuropsychopharmacol Biol Psychiatry 26: 651-661.

Näätänen R (2001). The perception of speech sounds by the human brain as reflected by the mismatch negativity (MMN) and its magnetic equivalent (MMNm). Psychophysiology 38: 1-21.

Nakagome K, Ichikawa I, Kanno O, Akaho R, Suzuki M, Takazawa $S$ et al (1998). Overnight effects of triazolam on cognitive function: an event-related potentials study. Neuropsychobiology 38: $232-240$.

Nichols JM, Martin F (1993). P300 in heavy social drinkers: the effect of lorazepam. Alcohol 10: 269-274.

Pekkonen E (2000). Mismatch negativity in aging and in Alzheimer's and Parkinson's diseases. Audiol Neuro-otol 5: 216-224.

Pekkonen E, Hirvonen J, Ahveninen J, Kähkönen S, Kaakkola S, Huttunen J et al (2002). Memory-based comparison process not attenuated by haloperidol: a combined MEG and EEG study. Neuroreport 13: 177-181.

Pekkonen E, Hirvonen J, Jääskeläinen IP, Kaakkola S, Huttunen J (2001). Auditory sensory memory and the cholinergic system: implications for Alzheimer's disease. Neuroimage 14: 376-382.

Picton T, Hillyard S, Galambos R (1976). Habituation and attention in the auditory system. In: Keidel W, Neff W (eds). Handbook of Sensory Physiology $V$ Auditory System. Springer: Berlin. pp 343-389.

Post RB, Chaderjian MR, Maddock RJ, Lott LA (1997). Effects of lorazepam on the distribution of spatial attention. Exp Clin Psychopharmacol 5: 143-149.

Riekkinen Jr P, Paakkonen A, Karhu J, Partanen J, Soininen H, Laakso $\mathrm{M}$ et al (1997). THA disrupts mismatch negativity in Alzheimer disease. Psychopharmacology (Berl) 133: 203-206.

Rinne T, Alho K, Ilmoniemi RJ, Virtanen J, Näätänen R (2000). Separate time behaviors of the temporal and frontal mismatch negativity sources. Neuroimage 12: 14-19.

Rockstroh B, Elbert T, Lutzenberger W, Altenmüller E (1991). Effects of the anticonvulsant benzodiazepine clonazepam on event-related brain potentials in humans. Electroencephalogr Clin Neurophysiol 78: 142-149.

Roeser R, Price LL (1969). Effects of habituation on the auditory evoked response. J Aud Res 9: 306-313.

Rosburg T (2004). Effects of tone repetition on auditory evoked neuromagnetic fields. Clin Neurophysiol 115: 898-905.

Rosburg T, Haueisen J, Sauer H (2002). Habituation of the auditory evoked field component $\mathrm{N} 100 \mathrm{~m}$ and its dependence on stimulus duration. Clin Neurophysiol 113: 421-428.

Rosburg T, Kreitschmann-Andermahr I, Nowak H, Sauer H (2000). Habituation of the auditory evoked field component $\mathrm{N} 100 \mathrm{~m}$ in male patients with schizophrenia. J Psychiatr Res 34: 245-254.

Rosburg T, Kreitschmann-Andermahr I, Ugur T, Haueisen J, Sauer $\mathrm{H}$ (2001). Mismatch fields (MMF) to deviants of different features. In: Nenonen J, Ilmoniemi RJ, Katila T (eds). Biomag 2000, Proceedings of 12 International Conference on Biomagnetism. Helsinki University of Technology, Espoo. pp 70-73.

Ruijter J, De-Ruiter MB, Snel J (2000a). The effects of caffeine on visual selective attention to color: an ERP study. Psychophysiology 37: 427-439.

Ruijter J, De-Ruiter MB, Snel J, Lorist MM (2000b). The influence of caffeine on spatial-selective attention: an event-related potential study. Clin Neurophysiol 111: 2223-2233.

Ruijter J, Lorist MM, Snel J, De-Ruiter MB (2000c). The influence of caffeine on sustained attention: an ERP study. Pharmacol Biochem Behav 66: 29-37.

Schwender D, Klasing S, Madler C, Poppel E, Peter K (1993). Effects of benzodiazepines on mid-latency auditory evoked potentials. Can J Anaesth 40: 1148-1154.

Semlitsch HV, Anderer P, Saletu B (1995). Acute effects of the anxiolytics suriclone and alprazolam on cognitive information processing utilizing topographic mapping of event-related brain potentials (P300) in healthy subjects. Eur J Clin Pharmacol 49: 183-191.

Serra JM, Escera C, Sanchez-Turet M, Sanchez-Sastre J, Grau C (1996). The H1-receptor antagonist chlorpheniramine decreases the ending phase of the mismatch negativity of the human auditory event-related potentials. Neurosci Lett 203: 77-80.

Sinton CM, McCullough JR, Ilmoniemi RJ, Etienne PE (1986). Modulation of auditory evoked magnetic fields by benzodiazepines. Neuropsychobiology 16: 215-218.

Smolnik R, Molle M, Fehm HL, Born J (1999). Brain potentials and attention after acute and subchronic intranasal administration of ACTH 4-10 and desacetyl-alpha-MSH in humans. Neuroendocrinology 70: 63-72.

Smolnik R, Pietrowsky R, Fehm HL, Born J (1998). Enhanced selective attention after low-dose administration of the benzodiazepine antagonist flumazenil. J Clin Psychopharmacol 18: 241-247.

Stephenson CME, Suckling J, Dirckx SG, Ooi C, McKenna PJ, Bisbrown-Chippendale R et al (2003). GABAergic inhibitory mechanisms for repetition-adaptivity in large-scale brain systems. Neuroimage 19: 1578-1588.

Study RE, Barker JL (1981). Diazepam and (-)-pentobarbital: fluctuation analysis reveals different mechanisms for potentiation of gamma-aminobutyric acid responses in cultured central neurons. Proc Natl Acad Sci USA 78: 7180-7184.

Tulen JH, Man in't Veld AJ. (1998). Noninvasive indices of autonomic regulation after alprazolam and lorazepam: effects on sympathovagal balance. J Cardiovasc Pharmacol 32: 183-190.

Umbricht D, Javitt D, Novak G, Bates J, Pollack S, Lieberman J et al (1998). Effects of clozapine on auditory event-related potentials in schizophrenia. Biol Psychiatry 44: 716-725.

Umbricht D, Javitt D, Novak G, Bates J, Pollack S, Lieberman J et al (1999). Effects of risperidone on auditory event-related potentials in schizophrenia. Int J Neuropsychopharmcol 2: 299-304.

Umbricht D, Koller R, Vollenweider FX, Schmid L (2002). Mismatch negativity predicts psychotic experiences induced by NMDA receptor antagonist in healthy volunteers. Biol Psychiatry 51: 400-406.

Umbricht D, Schmid L, Koller R, Vollenweider FX, Hell D, Javitt DC (2000). Ketamine-induced deficits in auditory and visual context-dependent processing in healthy volunteers: implications for models of cognitive deficits in schizophrenia. Arch Gen Psychiatry 57: 1139-1147.

Umbricht D, Vollenweider FX, Schmid L, Grubel C, Skrabo A, Huber $\mathrm{T}$ et al (2003). Effects of the 5-HT(2A) agonist psilocybin on mismatch negativity generation and AX-Continuous Performance Task: implications for the neuropharmacology of cognitive deficits in schizophrenia. Neuropsychopharmacology 28: $170-181$.

Velden M (1982). Die Signalentdeckungstheorie in der Psychologie. Kohlhammer: Stuttgart. pp 85.

Waberski TD, Kreitschmann-Andermahr I, Kawohl W, Darvas F, Ryang Y, Gobbele R et al (2001). Spatio-temporal source imaging reveals subcomponents of the human auditory mismatch negativity in the cingulum and right inferior temporal gyrus. Neurosci Lett 308: 107-110.

Woldorff MG, Gallen CC, Hampson SA, Hillyard SA, Pantev C, Sobel D et al (1993). Modulation of early sensory processing in human auditory cortex during auditory selective attention. Proc Natl Acad Sci USA 90: 8722-8726.

Woldorff MG, Hillyard SA, Gallen CC, Hampson SR, Bloom FE (1998). Magnetoencephalographic recordings demonstrate attentional modulation of mismatch-related neural activity in human auditory cortex. Psychophysiology 35: 283-292.

Wolff C, Schröger E (2001). Activation of the auditory pre-attentive change detection system by tone repetitions with fast stimulation rate. Brain Res Cogn Brain Res 10: 323-327. 